Didáctica Geográfica n 22, 2021, pp. 123-144

DOI: https://doi.org/10.21138/DG.609

ISSN electrónico: 2174-6451

\title{
LA EVALUACIÓN EN LA REVISTA DIDÁCTICA GEOGRÁFI- CA: UNA REVISIÓN BIBLIOMÉTRICA
}

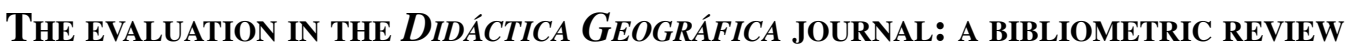

L'ÉVALUATION DANS LA REVUE DIDÁCTICA GEOGRÁFICA: UNE RÉVISION BIBLIOMÉTRIQUE

Clara Moya Parreño

Universidad de Alicante

cmp112@alu.ua.es

Rafael Sebastiá Alcaraz

Universidad de Alicante

rafael.sebastia@ua.es

Emilia María Tonda Monllor

Universidad de Alicante

emilia.tonda@ua.es

Recibido: 24/03/2021

Aceptado: 12/05/2021

\section{Resumen:}

La revisión bibliométrica que se realiza en esta aportación se centra en un análisis de la revista Didáctica Geográfica y en el tema de la evaluación. La delimitación temporal de la investigación corresponde a los cinco últimos años, aunque realmente aborda un periodo más amplio que afecta a la denominada Segunda Época (1996-...) de la revista. La principal aportación de este artículo es avanzar en un componente esencial tanto en la investigación como en la enseñanza de la Geografía: la evaluación. Los resultados del análisis han puesto de manifiesto la escasez de investigaciones relacionadas con la 
evaluación en la enseñanza de la geografía, y lo que es más significativo, la parquedad de criterios de evaluación en las experiencias y producciones didácticas de Geografía. Con esta investigación se propone la necesidad de detallar la evaluación y no reducir la misma, como suele ser frecuente, a los criterios que se derivan de las calificaciones (notas) académicas, u obviarla.

\section{Palabras clave:}

Evaluación; Didáctica; Geografía; revisión bibliométrica; Didáctica Geográfica.

\section{Abstract:}

The bibliometric review carried out in this contribution focuses on an analysis of the Didáctica Geográfica journal and on the subject of evaluation. The temporal delimitation of this work corresponds to the last five years, although it really addresses a broader period that affects the so-called Second Epoch (1996-...) of the journal. The main contribution of this article is to advance in an essential component both in the investigation and in the teaching of geography: the evaluation. The results of the analysis have revealed the scarcity of research related to evaluation in the teaching of geography, and what is more significant, the paucity of evaluation criteria in the didactic experiences and productions of Geography. With this result, the need to detail the evaluation and not reduce it many times to the criteria derived from the academic grades (grades), or ignore it, is proposed.

\section{KEYWORDS:}

Evaluation; Didactic; Geography; bibliometric review; Didáctica Geográfica.

\section{RÉSUMÉ:}

La révision bibliométrique réalisée dans cette contribution porte sur une analyse de la revue Didáctica Geográfica et sur le sujet de l'évaluation. La délimitation temporelle de l'étude de recherche correspond aux cinq dernières années, bien qu'elle aborde en réalité une période plus large qui affecte la soi-disant Seconde Epoque (1996-...) de la revue. La principale contribution de cet article est d'avancer dans une composante essentielle à la fois dans la recherche et dans l'enseignement de la géographie: l'évaluation. Les résultats de l'analyse ont révélé la rareté des recherches liées à l'évaluation dans l'enseignement de la géographie, et ce qui est plus significatif la rareté des critères d'évaluation dans les expériences et les productions didactiques de la géographie. Avec ce résultat, il est proposé de détailler l'évaluation et de ne pas la réduire souvant aux critères dérivés des notes académiques (notes), ou de l'ignorer. 


\section{MoTS-CLÉS:}

Évaluation; Didactique; Géographie; révision bibliométrique; Didáctica Geográfica.

\section{INTRODUCCIÓN}

Esta investigación tiene como objetivo principal analizar los cinco últimos años de la revista Didáctica Geográfica para examinar cuántos artículos tratan sobre evaluación. El tema es de interés puesto que apenas se encuentran investigaciones científicas que aborden la cuestión de la evaluación y, menos todavía, si se concreta en el ámbito de la enseñanza de la Geografía. Sin embargo, la evaluación es la base tanto del proceso de enseñanza aprendizaje como de la investigación, y contribuye a la mejora de la eficacia y eficiencia del sistema educativo.

La investigación se ha centrado en aquellos artículos en los que se incluyen propuestas o materiales didácticos. El objetivo que se persigue es conocer primero si se incluye el proceso de evaluación, segundo descubrir la forma en la que se practica y finalmente detallar los criterios que aplican los investigadores para evaluar sus propuestas didácticas. Otro objetivo de esta revisión ha sido dar a conocer el tema de la evaluación en la Didáctica de la Geografía y, si procede, avanzar en esta línea de investigación.

La presente aportación incluye inicialmente, una justificación del por qué se ha seleccionado la revista Didáctica Geográfica para realizar el análisis. A continuación, se expone la metodología utilizada y posteriormente, el análisis de los datos que se divide en dos apartados, uno sobre aspectos formales, y otro sobre los contenidos estructurales. Finalmente, se exponen las conclusiones obtenidas.

\section{FUENTES DOCUMENTALES PARA LA INVESTIGACIÓN}

La presente aportación se ha centrado en el análisis y evaluación de una revista esencial para la investigación e innovación en la Geografía y su didáctica, y la única en España especializada en esta temática, con una dilatada trayectoria que se remonta a 1977. A ella se puede acceder tanto en soporte papel, como de manera on-line, a través del siguiente enlace: $\underline{w w w . d i d a c t i c a g e o g r a f i c a . c o m}$

Sobre el objetivo de la investigación que se propone cabe advertir que es nuevo y que no cuenta con antecedentes. No obstante, anteriormente se han llevado a cabo análisis bibliométricos centrados en la revista Didáctica Geográfica (Sebastiá y Tonda, 2011; Jerez y Córdoba, 2016; López y Martínez, 2016). Otros ejemplos de análisis bibliométrico centrados en otras fuentes documentales más recientes son los de GómezCarrasco, López-Facal y Rodríguez-Medina (2019) y Morote (2020). Otro antecedente de investigación sobre la evaluación, pero centrada en el alumnado y en los instrumentos, 
es el de García (1988). Posteriormente, hubo un congreso de didáctica de las ciencias sociales sobre la evaluación en Geografía e Historia (Prieto, Valera y Gomariz, 2011) pero una vez más estuvo centrado en el alumnado. La novedad en esta reunión científica fue la irrupción de las competencias que se relacionaron con la evaluación (Valera, Gomariz y Prieto, 2011).

Por otro lado, se eligió la revista de Didáctica Geográfica principalmente porque sus objetivos están encaminados a la mejora de la enseñanza de la Geografía. Y, este objetivo está estrechamente vinculado al que se pretende conseguir con la revisión que se presenta a continuación.

\section{MÉTODO UTILIZADO}

La definición del método de análisis no es una labor sencilla, pues la falta de referentes que globalicen los campos o ítems de estudio hace que la concreción de las áreas a evaluar sea difícil de legitimar. El procedimiento seguido ha sido más analítico que interpretativo. Sebastiá y Tonda (2014) señalan que "los referentes documentales consultados sobre procedimientos de análisis bibliográficos muestran que cada investigador selecciona los criterios según la finalidad que persigue, y que no existe un modelo único que se pueda generalizar" (p. 108). No obstante, es necesario dotar de credibilidad a la investigación, por tanto, los ítems que se van a evaluar han sido expuestos anteriormente en diferentes foros científicos y no han sido cuestionados hasta la fecha. En la concreción de los campos utilizados para analizar las propuestas didácticas se han considerado los establecidos por Santos (1988). Por otro lado, los ítems utilizados para la evaluación de los materiales y experiencias didácticas se ajustan al método DAFO (Debilidades, Amenazas, Fortalezas y Oportunidades).

En cuanto a la delimitación temporal, queda definida al intervalo comprendido entre los años 2016 y 2020 (ambos inclusive), correspondientes a los números 17, 18, 19, 20 y 21 de la revista, ajustándose al criterio de considerar el último quinquenio que se utiliza en la investigación general de la producción científica en diferentes foros como por ejemplo Google académico. Como indica Bauman (2013) en esta sociedad líquida, la evaluación de una trayectoria profesional tiende a concentrarse en los últimos años de práctica profesional y se olvida lo que puede ser una amplia trayectoria laboral. De este modo, aunque la revista Didáctica Geográfica cuenta con una sólida trayectoria, el análisis ha buscado centrarse en los cinco últimos años.

\section{ANÁLISIS DE LA REVISTA Y LOS ARTÍCULOS SELECCIONADOS}

Una vez revisados los últimos números de la revista, se ha de señalar que no se ha encontrado ningún artículo relacionado directamente con la evaluación. Este dato 
permite inferir la nula atención que merece esta cuestión tan esencial en la enseñanza y en la investigación didáctica. Para contextualizar mejor la investigación, la consulta de la hemeroteca se extendió a toda la segunda época de la revista. De todo este periodo solamente se ha encontrado el artículo de Sande (2005) titulado Simulación y Evaluación. En él, la autora realiza una revisión de la literatura escrita hasta la fecha (2005) sobre la evaluación de los impactos educativos de las simulaciones.

Puesto que la situación encontrada ha sido la expuesta y este trabajo no deja de ser una revisión bibliométrica, se decidió ampliar la investigación a artículos en los que se expusieran propuestas, materiales o recursos didácticos. Así, se pretende analizar cómo es la evaluación realizada sobre ellos. Resultado de esta selección, con estos nuevos criterios, se recopilaron un total de 23 artículos.

\subsection{Análisis formal}

El análisis practicado ha tenido en cuenta tres aspectos formales que se consideran procedentes. En primer lugar, se ha estudiado la distribución temporal de los artículos (Figura 1).La información obtenida permite comprobar que el año que más publicaciones comprende ha sido el 2017 con ocho artículos y el mínimo de tres en 2019. Por tanto, se comprueba una amplia variación anual, y tendencia recesiva.

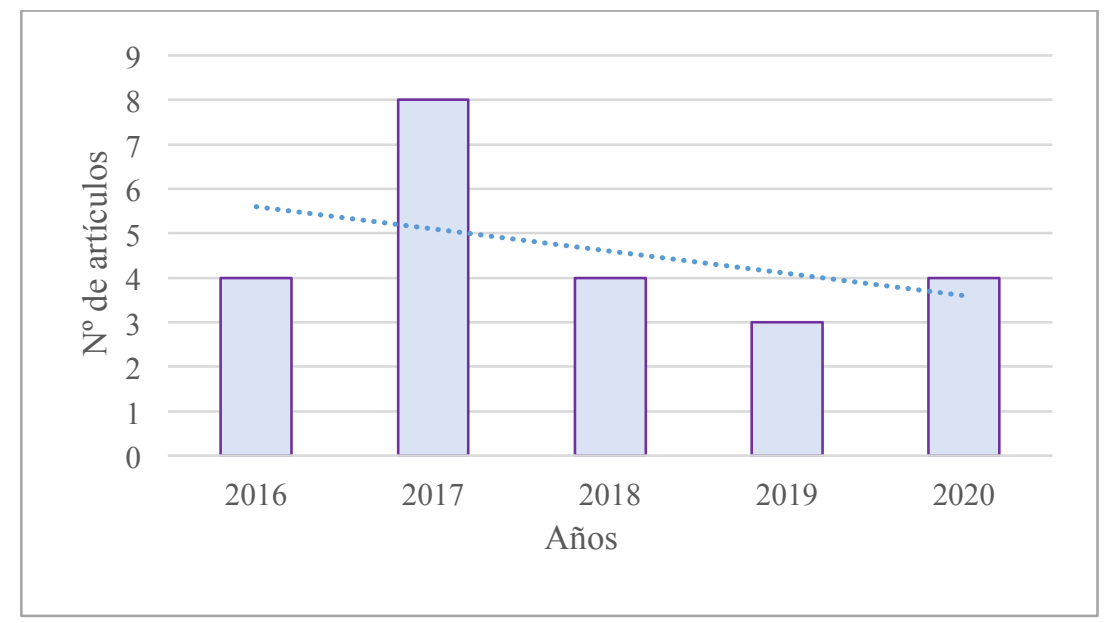

FiguRA 1. Número de artículos por año. Fuente: elaboración propia

En segundo lugar, se ha estudiado la autoría de los artículos desde el punto de vista del género. Los datos permiten inferir la ausencia de una distribución paritaria como se 
puede observar en la Figura 2 donde el mayor porcentaje corresponde a los hombres (74\%), lo que representa 34 autores del total de 46.

Los resultados obtenidos concuerdan con las cifras presentadas por el CSIC en su informe anual sobre investigadoras (2020). En este informe se pueden ver los datos de la distribución del personal investigador por sexo. Así, en la mayoría de los campos (postdoctoral, Ramón y Cajal, científicos titulares, investigadores científicos y profesores investigadores) el porcentaje de hombres es más elevado que el de mujeres. Además, este porcentaje va creciendo conforme vamos subiendo en la escala de puestos. El único campo en el que las mujeres tienen un porcentaje mayor es en el predoctoral (el escalón más bajo). No obstante, la diferencia es mínima, pues las mujeres tienen un $50,8 \%$ y los hombres un $49,2 \%$. Nada que ver con la mayor diferencia entre ambos sexos representada por el campo de los profesores investigadores en el que el 73,5\% son hombres y solamente el $26,5 \%$ mujeres.

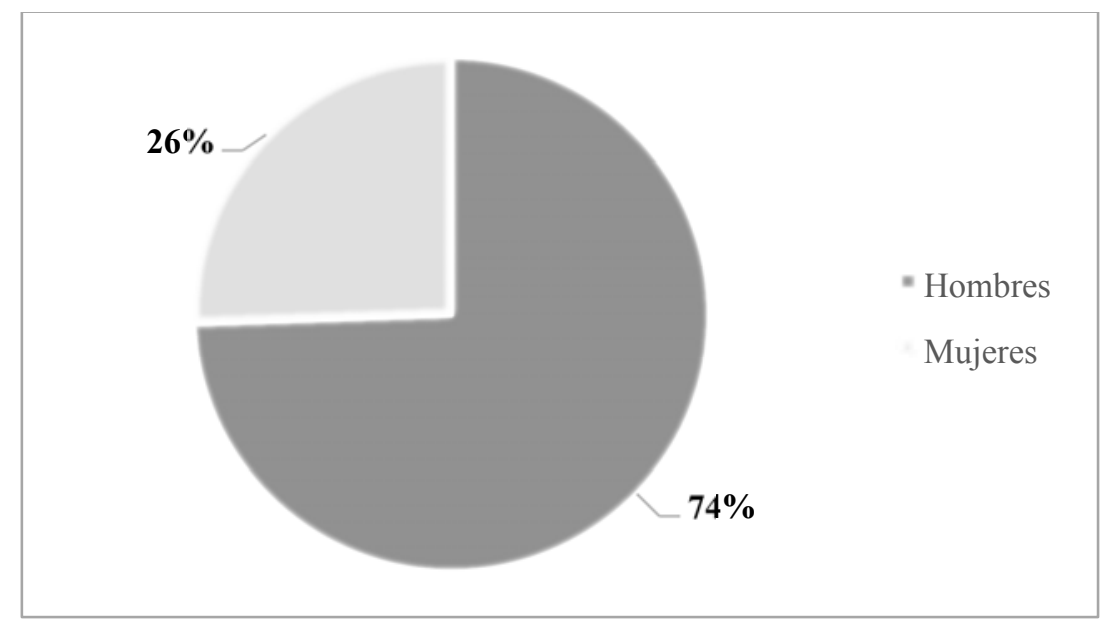

FiguRA 2. Autoría de los artículos, según su género. Fuente: elaboración propia

En tercer lugar, en relación con los autores, también se estudió el lugar de procedencia (Figura 3). Se ha querido saber de qué universidades españolas procedían los autores de los artículos. Una parte relevante (19,56\%) eran de la Universidad de Sevilla; 9 autores del total de 46. Tras ella, destaca la Universidad de Salamanca con 5 autores $(10,87 \%)$. En esta misma figura se puede comprobar la amplia dispersión territorial representada por 11 provincias españolas. La presencia internacional es más reducida pues sólo hay cinco autores de universidades extranjeras. En concreto, se contabilizan tres autores de Venezuela, uno de Argentina y uno de Brasil. Esto es muy interesante, pues es beneficioso 
que la investigación se enriquezca con aportaciones de diferentes contextos, y permita avanzar en generalizaciones más amplias.

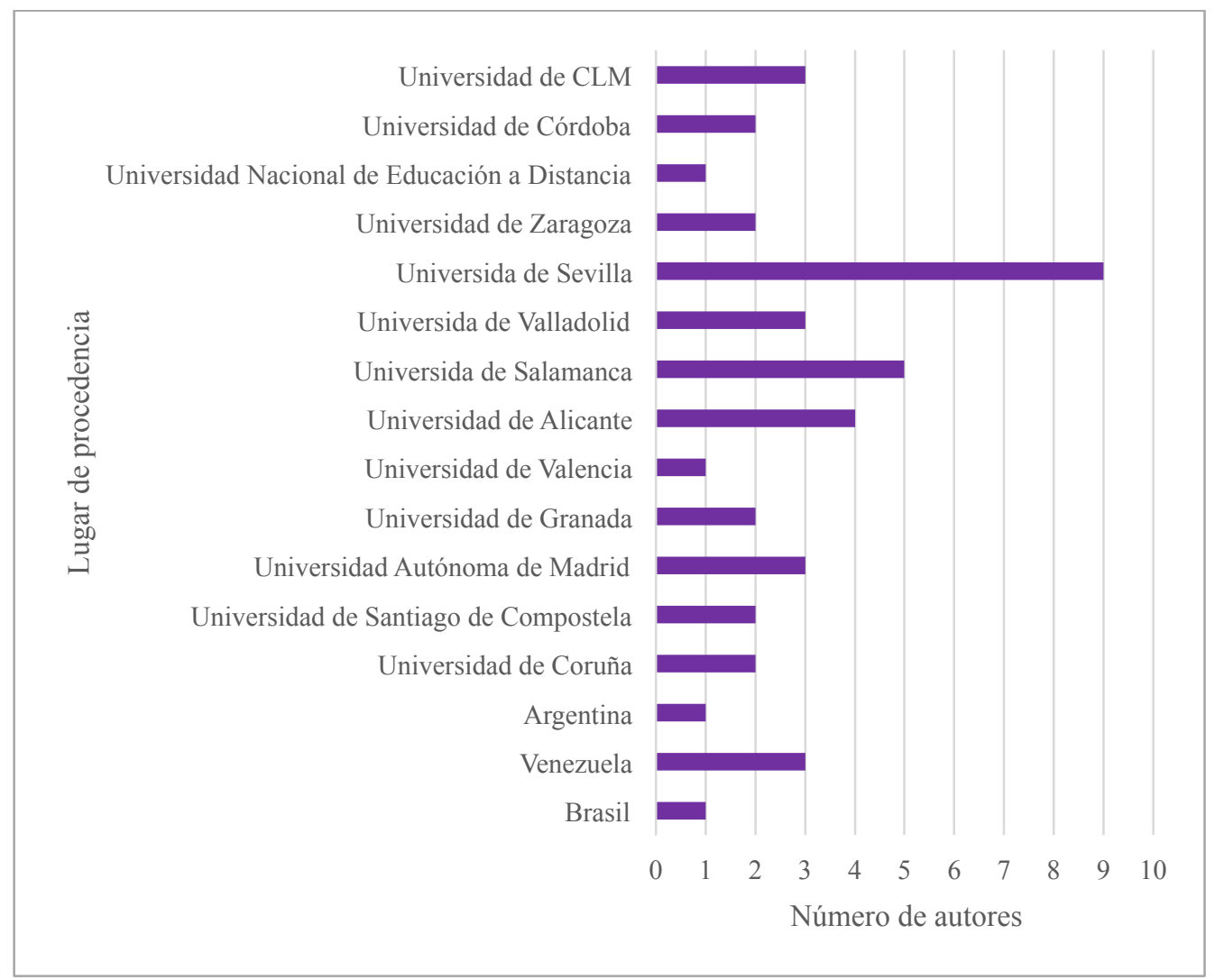

FiguRA 3. Autoría de los artículos según su procedencia. Fuente: elaboración propia

\subsection{Análisis estructural}

El primer aspecto que se ha querido señalar, por su interés en la investigación, es el nivel educativo sobre el que versan las propuestas o recursos didácticos recogidos en estos artículos (Figura 4). Así, se puede observar que los niveles de Educación Primaria, Educación Secundaria y Universidad, están igualados con un total de 6 artículos cada uno. La Educación Infantil es la que ofrece una menor consideración ya que solo hay un artículo. 
Por otro lado, es cierto que hay documentos que no indican el nivel al que se dirigen sus propuestas didácticas, bien porque consideran que se pueden llevar a cabo en todos o casi todos los niveles educativos, o bien porque directamente no hacen referencia alguna al nivel en el que se puede aplicar ese recurso o propuesta.

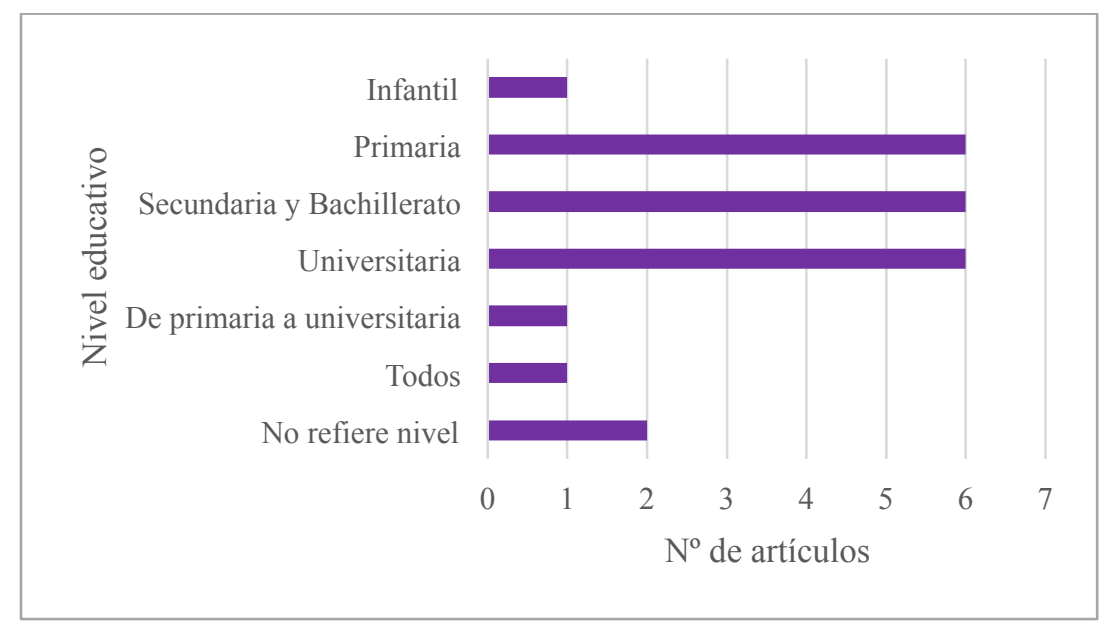

FIgURA 4. Nivel educativo al que van dirigidos los artículos. Fuente: elaboración propia

Llegados a este punto, se hizo necesario clasificar los artículos según trataran de propuestas didácticas, materiales o recursos. Como se puede observar en la Figura 5, no se encontraron artículos sobre materiales didácticos. La mayoría de las aportaciones seleccionadas correspondían a recursos didácticos y a propuestas de cómo utilizarlos en las aulas (87\%), es decir, 20 de los 23 artículos.

Es necesario señalar que materiales y recursos didácticos son conceptos diferentes y que no se pueden considerar sinónimos. Los materiales didácticos son creados con la finalidad explicita de enseñar y ser utilizados en las aulas para ser aplicados exclusivamente en la docencia. Los recursos didácticos se crean con una intención inicial distinta a la enseñanza, pero pueden ser utilizados para enseñar.

El ítem o categoría de propuestas didácticas representa el 13\% del total; esto es 3 artículos. Estas propuestas didácticas pueden servir de referencia u orientación para conocer otras perspectivas diferentes a las que nosotros hubiéramos recurrido, y avanzar en la generalización. 


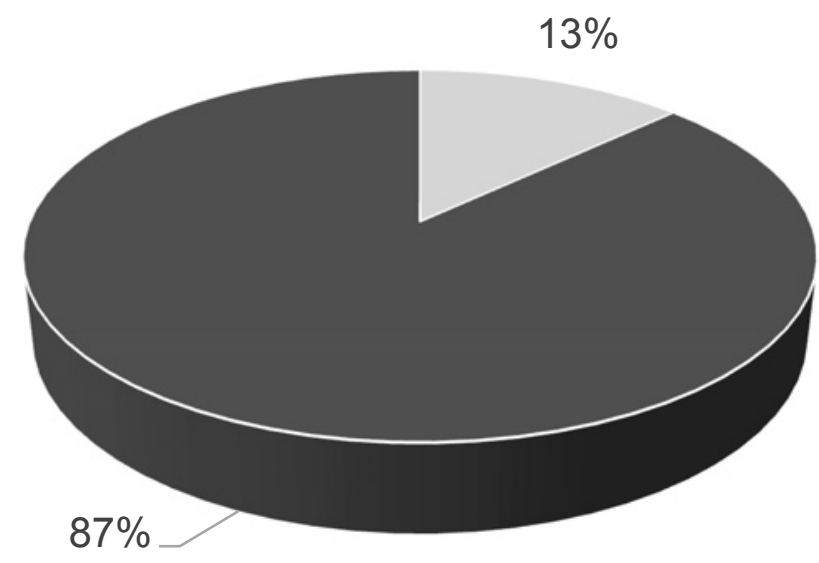

\section{- Propuestas - Recursos didácticos}

FIGURA 5. Porcentaje de recursos y propuestas didácticas. Fuente: elaboración propia

El análisis de la categoría de recursos didácticos avanzó en detalle al considerar también su tipología. Así, quedaron establecidas 6 subcategorías en base a los 20 artículos relacionados con los recursos didácticos: mapas, itinerarios/salidas de campo, museos, geoparques, TICS y pintura (Figura 6). La tipología más representada es la de los itinerarios/salidas de campo, con 12 artículos. Este recurso didáctico ha resultado ser el elemento más frecuente en el periodo analizado, y coincide con una referencia clásica en la enseñanza de la Geografía.

La subcategoría de recursos TIC ocupa el segundo puesto con 4 artículos. Esto sí es de extrañar, pues se considera que las nuevas tecnologías están muy presentes en las aulas tanto de Educación Primaria (6-11 años), como de Secundaria-Bachillerato (1218 años), o de Universidad. Por tanto, si hubiera una vinculación real entre la práctica docente y la investigación educativa este tipo de recursos deberían ser mucho más investigados. Sin embargo, esto demuestra la falta de vinculación de la investigación con la realidad del aula.

Los recursos menos tratados, aunque no por ello menos interesantes, son los museos, los geoparques, la pintura y los mapas. De todos ellos únicamente se ha encontrado un artículo de cada uno. 


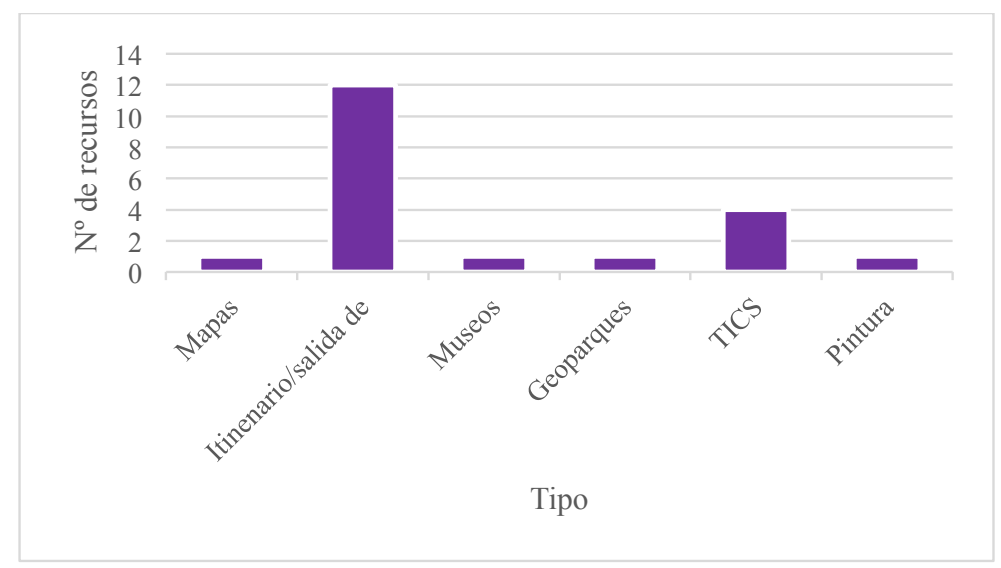

FiguRA 6. Tipos de recursos didácticos. Fuente: elaboración propia

Para profundizar en el tema de la evaluación, como ya se ha indicado, la muestra se ha ampliado con aquellas aportaciones que hacen referencia a la evaluación en sus propuestas y recursos didácticos de forma explícita (Figura 7). Solamente en el 35\% de estos artículos, es decir 8 artículos, mencionan la evaluación. Este dato permite una vez más poner de manifiesto la ausencia de la evaluación en la investigación e innovación que se está realizando en la enseñanza de la Geografía, y al mismo tiempo confiere valor a la investigación que se presenta. Pues la finalidad que se persigue es la de incorporar la evaluación a este proceso. La escasa limitación de artículos como se ha indicado, 8 , no debe ir en detrimento de las conclusiones que se presentan, sino al contrario, de la necesidad de incorporar este elemento en nuestras aportaciones.

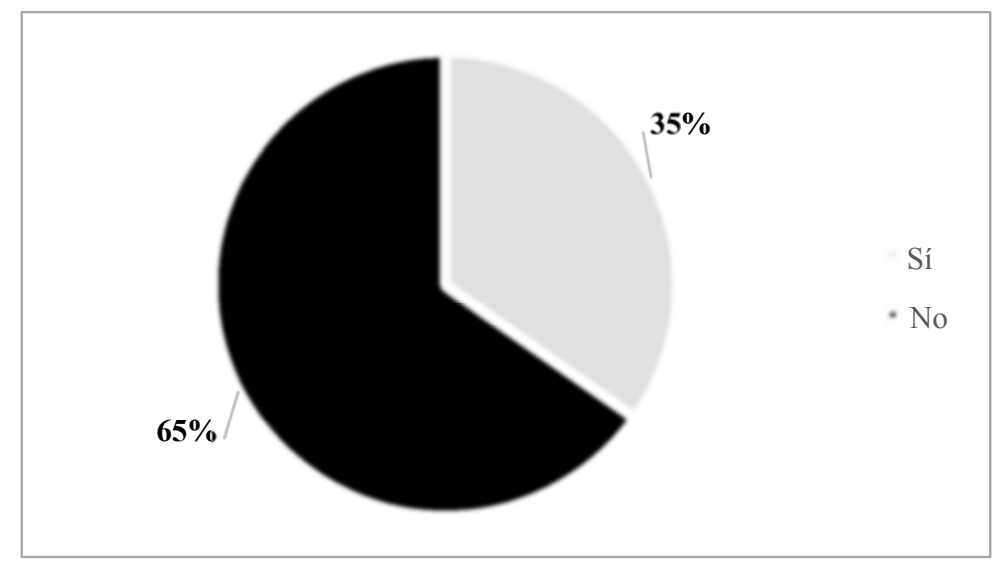

FiguRA 7. Porcentaje de artículos que incluyen o no la evaluación. Fuente: elaboración propia 
El análisis de la evaluación en esta muestra se ha realizado de forma cualitativa. No obstante, para sintetizar la información y facilitar la comunicación se ha elaborado una plantilla orientativa (Tabla 1). En ella, se emplean ocho ítems concretos elegidos de acuerdo con las características que debería tener una evaluación completa y apropiada de un recurso didáctico. Como se ha indicado anteriormente, algunos de los ítems con los que se evalúan los recursos didácticos ya fueron propuestos por Sebastiá y Tonda (2016). Otros, se han establecido en base al artículo de Santos (1988). De este modo, el primer ítem sirve para identificar el recurso. El segundo, delimita el nivel educativo al que se dirige (Educación Infantil, Primaria, Secundaria, Bachillerato y Universidad). El tercero pretende establecer si los autores han considerado la evaluación de los objetivos valorando el nivel de consecución de los mismos. La identificación de los objetivos suele ser relativamente fácil pues están redactados en infinitivo. El cuarto, valora si han evaluado los contenidos conceptuales, procedimentales y actitudinales (si han sido suficientes, apropiados, etc.). El quinto analiza si se evalúa el nivel de adecuación de las actividades propuestas para utilizar el recurso. El sexto y séptimo ítem se apoyan en el método DAFO: en el sexto se analizan las debilidades (D) y amenazas (A) y en el séptimo las fortalezas $(\mathrm{F})$ y oportunidades $(\mathrm{O})$ de la evaluación propuesta por los autores. Finalmente, el octavo ítem se refiere a si han realizado una autoevaluación.

\section{Recurso didáctico}

Nivel educativo

Objetivos

Contenidos

Actividades

D-A

F-O

Autoevaluación

TABLA 1. Plantilla para orientar el análisis de la evaluación de recursos. Fuente: elaboración propia

Una vez analizados los artículos sobre recursos didácticos, se pasará a estudiar un documento que versa sobre la propuesta metodológica. Para guiar esta parte del análisis también se ha establecido una plantilla. En este caso, se han mantenido los ítems anteriores añadiendo dos más: uno para analizar si los materiales con los que trabajan en 
la propuesta son evaluados y otro para la metodología que emplean. Así, la plantilla ha quedado como se muestra en la Tabla 2.

\section{Propuesta didáctica}

Nivel educativo

Objetivos

Contenidos

Actividades

Materiales

Metodología

D-A

F-O

Autoevaluación

TABLA 2. Plantilla para orientar el análisis de la evaluación de propuestas. Fuente: elaboración propia

\section{RESULTADOS DEL ANÁLISIS}

Los resultados del análisis se separan en función de los artículos que exponen recursos didácticos y los que exponen propuestas didácticas. En primer lugar, se trabajará con aquellos que tratan recursos didácticos. Para tener una visión más clara de todos ellos, se ha elaborado la Tabla 3 con los datos de estos artículos.

El análisis sobre los recursos didácticos que se presenta sigue el orden alfabético de la Tabla 3.

En primer lugar, García, Villar, Fraile, Sánchez y Márquez (2018) exponen una salida de campo, concretamente la denominan "la Salida Itinerante de Geografía". Según estos docentes se trata de una experiencia académica transversal en la que profesores y alumnos universitarios recorrieron a pie durante varios días seguidos un espacio de carácter natural y rural. Esta salida tuvo como objetivo analizar las principales claves territoriales del ámbito recorrido. 


\section{Artículos relacionados con recursos didácticos}

Autor/es Título Año $\mathbf{N}^{\mathbf{0}}$ Páginas

1 García, Villar, Fraile, Se hace geografía al andar: la salida de campo 201819 103-125 Sánchez y Márquez itinerante y senderista.

2 Labarca, Barreto y $\quad$ Potencialidades geográficas de la Laguna de las 201819 127-148

Bernal Peonías (Venezuela) como museo natural para la enseñanza de la geografía física.

3 Labarca y Gouveia Los procesos exogenéticos: una geo-ruta 202021 175-201 didáctica por la costa occidental del lago de Maracaibo (Venezuela).

4 López y Peral Las vías verdes: escenario para trabajar el $2017 \quad 18$ 171-192 medio rural en educación primaria.

5 Morote El parque inundable "La Marjal" de Alicante 201718 211-230 (España) como propuesta didáctica para la interpretación de los espacios de riesgo de inundación

6 Morote y Hernández Propuesta de un recurso didáctico para la 201920 95-121 interpretación del paisaje. La salida de campo en la antigua Laguna de Villena (Alicante).

7 Najarro y Maroto El desarrollo de las competencias sociales: 201920 123-152 itinerario didáctico por los restos fenicio de Almuñécar (Granada).

TABLA 3. Artículos sobre recursos didácticos en la revista Didáctica Geográfica que incluyen la evaluación. Fuente: elaboración propia

La mejor parte de su evaluación fue la auto-evaluación exhaustiva a través del propio artículo. La actividad recoge, un proceso de coevaluación, pues implementan una encuesta a los alumnos/as que han participado para que realicen la valoración a partir de su experiencia. La evaluación incorpora propuestas para mejorar el itinerario realizado.

La reflexión final es que es una experiencia beneficiosa según infieren del alto grado de satisfacción manifestado por el alumnado. En la evaluación práctica se echa en falta que no se considere si se han conseguido los objetivos propuestos; si los contenidos han sido suficientes y adecuados a los objetivos y si se han logrado a través de las actividades propuestas. Del mismo modo, tampoco evalúan si las actividades han sido las adecuadas o si hubiese que modificarlas.

Así pues, realizan una buena evaluación sobre los beneficios de utilizar el recurso de la salida de campo relacionados con la convivencia académica, las metodologías docentes, el vínculo de la geografía con la salud y la actividad deportiva, la idoneidad de ciertos 
territorios "más transversales", el proceso de retroalimentación flexible y la integración curricular. La efectividad real que este recurso tiene en el ámbito académico sobre cómo mejorar el proceso de enseñanza-aprendizaje y el por qué no se han tenido en cuenta.

En segundo lugar, Labarca, Barreto y Bernal (2018), proponen una visita guiada a la Laguna de las Peonías (Venezuela) para utilizar sus potencialidades didácticas como museo natural para la enseñanza de la geografía física. Estos docentes realizan una propuesta muy completa en el uso de este recurso didáctico. El itinerario se desarrolla en cinco paradas diferentes y para cada una de ellas se propone una actividad concreta de evaluación. Estas actividades son muy adecuadas porque a través de ellas se evalúa si el alumnado ha conseguido los objetivos propuestos, si ha adquirido los contenidos y si las actividades que se han realizado han sido adecuadas para ello. Pero, desde el punto de vista de la presente investigación se considera que quedan dos cuestiones pendientes.

La primera y principal es que no indican a qué edad va dirigido este recurso. Por tanto, es un poco complejo implementar una propuesta sobre un recurso si no se conoce el nivel educativo al que va dirigido. La segunda es que no se realiza una evaluación del recurso como tal. Solamente se evalúa al alumnado y la evaluación debería ir dirigida a todos los participantes indistintamente. Y, por supuesto, falta la evaluación del recurso en sí.

En tercer lugar, Labarca y Gouveia (2020) presentan el recurso de una geo-ruta didáctica por la costa occidental del Lago de Maracaibo (Venezuela) con el objetivo de que el estudiante alcance a descubrir y describir procesos exogenéticos en el campo.

Estos autores, al igual que los del artículo anterior, proponen actividades de evaluación completas para conocer la consecución de los objetivos, la adquisición de los contenidos y la eficacia de las actividades. Además, añaden un punto fuerte, pues plantean actividades que permiten una evaluación tanto cuantitativa como cualitativa. Esto es importante ya que no solamente hay que utilizar una cuantificación para evaluar a los alumnos/as; los aspectos cualitativos también son muy importantes.

La cuestión para revisar es que igualmente la evaluación es unidireccional, es decir, profesor - alumno. Otro punto por considerar es la ausencia de la evaluación del propio recurso.

En cuarto lugar, López y Peral (2017), introducen una salida de campo relacionada con las vías verdes. Concretamente, definen las vías verdes como un escenario adecuado para trabajar el medio rural en la educación primaria y realizan una propuesta de trajo utilizando el recurso de la Vía Verde de la Campiña, situada entre las provincias de Córdoba y Sevilla.

El artículo presenta una evaluación muy completa. En primer lugar, añade la evaluación inicial del alumnado estudiando cuáles son sus conocimientos e ideas previas sobre el tema que se va a tratar. Después, señala que se realizará una autoevaluación de la 
actividad teniendo en cuenta tanto los objetivos didácticos como los objetivos docentes. Este es un punto que hasta el momento no se había visto reflejado en ningún otro artículo.

Por otro lado, evalúa si el alumnado ha adquirido los conocimientos y propone actividades de refuerzo para asentarlos en el caso de que no se haya producido un aprendizaje significativo. Además, señala que, desde el punto de vista docente, será necesario realizar una evaluación procesual de la actividad para plantear modificaciones y/o correcciones posteriores con la intención de mejorar tanto las actividades (si fuese necesario) como los aspectos contextuales.

Así, la evaluación queda bastante completa. No obstante, la principal debilidad radica en que todo lo expuesto anteriormente queda reflejado en el artículo de forma global. No se especifica cómo se van a llevar a cabo cada uno de los actos evaluativos. Igualmente, aunque es bueno que haya autoevaluación por parte del docente en cuanto a los objetivos, falta la evaluación en la dirección alumno - profesor y la evaluación de los beneficios de utilizar este recurso concreto y no otro.

En quinto lugar, Morote (2017) presenta el recurso del parque inundable "La Marjal" de Alicante para trabajar la interpretación de los espacios de riesgo de inundación. Se trata de un tema trascendente pues su aportación no se dirige sólo a poner en evidencia las condiciones climáticas y los desastres que acompañan las lluvias torrenciales, que permite descubrir como la intervención responsable del ser humano puede paliar los daños y contribuir a un desarrollo sostenible.

En contraposición al artículo anterior, la evaluación realizada por Morote incluye opciones de actividades evaluativas para todos los niveles educativos; incluso divide las actividades de evaluación de la etapa de primaria en dos bloques (de $1^{\circ}$ a $3^{\circ}$ curso y de $4^{\circ}$ a $6^{\circ}$ curso). No obstante, aparte de evaluar la adquisición de los contenidos por parte del alumnado, no realiza ningún otro tipo de evaluación.

En sexto lugar, Morote y Hernández (2019), proponen un recurso didáctico para la interpretación del paisaje: una salida de campo a la antigua Laguna de Villena (Alicante). En este artículo apenas se hace mención a la evaluación. No obstante, con el párrafo que le dedican se pueden sacar varias conclusiones sobre sus fortalezas y debilidades. Por un lado, exponen que la evaluación de la actividad se realizará a través de la entrega de una ficha de actividades que se da al alumnado antes de comenzar la salida de campo. Está bien que el alumnado sepa sobre qué se le va a evaluar, pero debería haber otro tipo de evaluación paralela a la ficha de actividades. No obstante, con estas actividades se evalúa si han conseguido el aprendizaje de los contenidos propuestos y las dificultades que han encontrado en las actividades. Por tanto, están evaluando la eficacia de las actividades propuestas. Por otro lado, estos investigadores no incluyen una evaluación del grado 
de consecución de los objetivos. Tampoco se realiza una evaluación bidireccional, autoevaluación, ni evaluación del recurso en sí.

Para terminar con este grupo de artículos, Najarro y Maroto (2019) exponen el itinerario didáctico por los restos fenicios de Almuñécar (Granada) con el fin de desarrollar las competencias sociales y cívicas en ciencias sociales. En este caso, la evaluación del alumnado se lleva a cabo tanto a través de un cuaderno de campo como de un producto final (elaboración de una carta reivindicativa). Esto es positivo puesto que a través de estas dos actividades evaluativas se considera si se han cumplido los objetivos, si los contenidos han sido adquiridos y la validez de las actividades queda reflejada a través del cuaderno de campo. Además, que se considere tanto el cuaderno de campo como un producto final significa que se está llevando a cabo una evaluación tanto del proceso de aprendizaje como de la culminación del mismo. Por otro lado, también enriquecen la evaluación ya que incluyen una rúbrica específica para valorar el grado en el que se ha conseguido desarrollar las competencias clave. No obstante, la evaluación sigue siendo en una sola dirección y no contemplan ni la autoevaluación ni la evaluación del recurso en sí.

Una vez analizados los artículos sobre recursos didácticos, se avanza hacia el siguiente punto que es considerar la propuesta metodológica. Del mismo modo, se ha creado una tabla resumen (Tabla 4) con la referencia sintetizada del artículo que se analiza a continuación.

\begin{tabular}{lllllll}
\hline Núm. & \multicolumn{5}{c}{ Artículos Propuestas Didácticas } \\
& Autor/es & \multicolumn{1}{c}{ Título } & Año & $N^{\boldsymbol{o}}$ & Páginas \\
\hline 8 & Lucendo & Propuesta de metodología docente para la & 2016 & 17 & $79-100$ \\
& & adaptación de materias de geografía descriptiva al \\
& EEES: el caso de la asignatura de Geografía de & & & \\
& Europa. & & \\
\hline
\end{tabular}

TABLA 4. Artículos sobre propuestas didácticas en la revista Didáctica Geográfica que incluyen la evaluación. Fuente: elaboración propia

La propuesta metodológica de Lucendo (2016) trata sobre un nuevo método llamado NEW (en inglés New Education in Works) aplicado a la asignatura de Geografía de Europa.

Cuando se establece una propuesta didáctica basada en una metodología concreta, es necesario hacer una evaluación exhaustiva para detallar los beneficios que aporta. En esta ocasión, Lucendo ha realizado una evaluación centrada en actividades evaluativas para constatar la consecución de los contenidos establecidos por parte del alumnado. De este modo resulta difícil conocer el alcance de la eficacia de la propuesta 
metodológica, y convendría incorporar una justificación del desarrollo de la misma y de los materiales utilizados.

A modo de resumen, se incluye la Tabla 5 con los criterios que se han ido mencionando, considerando y citando a lo largo del análisis. Para ello, se ha marcado con una cruz los criterios que cumplen cada uno de los artículos seleccionados. Así, esta tabla sirve para visualizar rápidamente los criterios identificados, presentar resultados y, al mismo tiempo, realizar una autoevaluación propia.

\begin{tabular}{|c|c|c|c|c|c|c|c|c|c|}
\hline \multirow[t]{2}{*}{ Criterios } & \multicolumn{7}{|c|}{ Recursos y propuestas } & \multicolumn{2}{|r|}{ Total } \\
\hline & 1 & 2 & 3 & 4 & 5 & 6 & 7 & 8 & \\
\hline Evaluación inicial (ideas y conocimientos previos) & & & & $\mathrm{x}$ & & & & & 1 \\
\hline Considera los centros de interés & & & & & & & $\mathrm{x}$ & & 1 \\
\hline Se indican objetivos & $\mathrm{x}$ & $\mathrm{x}$ & & $\mathrm{x}$ & & & $\mathrm{x}$ & $\mathrm{x}$ & 5 \\
\hline $\begin{array}{l}\text { Se comunica los objetivos al alumnado para su } \\
\text { evaluación }\end{array}$ & & & & & & $\mathrm{x}$ & & & 1 \\
\hline Se indican los contenidos & $\mathrm{x}$ & $\mathrm{x}$ & $\mathrm{x}$ & $\mathrm{x}$ & & & $\mathrm{x}$ & $\mathrm{x}$ & 6 \\
\hline $\begin{array}{l}\text { Los contenidos se desglosan en conceptuales, } \\
\text { procedimentales y actitudinales }\end{array}$ & $\mathrm{x}$ & & & & & & & & 1 \\
\hline $\begin{array}{l}\text { Se indican las fases de evaluación (inicial, formativa, } \\
\text { sumativa) }\end{array}$ & & & & $\mathrm{x}$ & & & $\mathrm{x}$ & $\mathrm{x}$ & 3 \\
\hline $\begin{array}{l}\text { La evaluación se apoya sólo en la calificación del } \\
\text { alumnado }\end{array}$ & & $\mathrm{x}$ & $\mathrm{x}$ & & $\mathrm{x}$ & $\mathrm{x}$ & $\mathrm{x}$ & $\mathrm{x}$ & 6 \\
\hline La evaluación tiene en cuenta la opinión del alumnado & $\mathrm{x}$ & & $\mathrm{x}$ & $\mathrm{x}$ & & & & & 3 \\
\hline $\begin{array}{l}\text { La evaluación combina los criterios del docente y del } \\
\text { discente }\end{array}$ & $\mathrm{x}$ & & & $\mathrm{x}$ & & & & & 2 \\
\hline $\begin{array}{l}\text { La evaluación incluye la valoración de material } \\
\text { utilizados }\end{array}$ & & & & $\mathrm{x}$ & & & & & 1 \\
\hline La evaluación propone cambios para mejorar & $\mathrm{x}$ & & & $\mathrm{x}$ & & & & & 2 \\
\hline Se incluye autoevaluación & $\mathrm{x}$ & & & $\mathrm{x}$ & & & & & 2 \\
\hline Se considera la eficacia y la eficiencia en la evaluación & $\mathrm{x}$ & & & & & $\mathrm{x}$ & & & 2 \\
\hline $\begin{array}{l}\text { Se comparan los resultados entre grupos de referencia y } \\
\text { control }\end{array}$ & & & & & & & & & 0 \\
\hline Se evalúa la práctica docente & & & & $\mathrm{x}$ & & & & & 1 \\
\hline $\begin{array}{l}\text { La evaluación no es únicamente cuantitativa, también es } \\
\text { cualitativa }\end{array}$ & & & $\mathrm{x}$ & $\mathrm{x}$ & & & & & 2 \\
\hline Hay metaevaluación & & & & & & & & & 0 \\
\hline Hay paraevaluación & & & & & & & & & 0 \\
\hline Total & 8 & 3 & 4 & 11 & 1 & 3 & 5 & 4 & 39 \\
\hline
\end{tabular}

TABLA 5. Criterios de evaluación para la comparación de los recursos didácticos y propuestas. Fuente: elaboración propia 
Finalmente, con la presente tabla se plantea la posibilidad de considerar otras categorías para la evaluación y fundamentación de las conclusiones, pues se indican los criterios que están más o menos presentes.

Como se puede observar, casi todos los ítems han tenido algún punto, exceptuando tres de ellos: la comparación de resultados entre grupos de referencia y control, la metaevaluación y la paraevaluación. De esta manera, queda patente que no se realizan comparaciones, evaluación de la evaluación, ni evaluaciones profundas que vayan más allá de los simples aspectos técnicos considerando la eficacia real de la propuesta o recurso implementado.

Por otro lado, los tres ítems más puntuados nos indican que, en primer lugar, normalmente se establecen los contenidos y los objetivos (algo lógico, pues son un pilar fundamental de las propuestas didácticas). En segundo lugar, en general la evaluación se realiza centrándose únicamente en las calificaciones del alumnado, lo que indica que no se están realizando evaluaciones de calidad, cuestión recogida en el cuarto punto de los Objetivos para el Desarrollo Sostenible (Educación de calidad).

\section{DISCUSIÓN Y CONCLUSIONES}

La presentación de las conclusiones finales se ha distribuido en tres puntos.

En primer lugar, la conceptualización pasa por la distinción entre evaluar y calificar. Si buscamos ambos términos en el Diccionario de la Real Academia Española, se puede ver que "calificar" se define como determinar cualidades o expresar un juicio sobre algo o alguien. Por el contrario, como atributos de criterio para el concepto de "evaluar" estaría: señalar, estimar, apreciar o incluso calcular el valor de algo. Por tanto, cuando se califica se está juzgando solo al alumnado y emitiendo un juicio de valor. Sin embargo, cuando se evalúa, los elementos que se consideran, las relaciones que se establecen y los procesos son bastante más amplios. La evaluación entendida también como emisión de juicios de valor a su vez ha de incluir y explicitar los criterios desde los que se realiza. Por esta razón, en esta aportación se presentan en una tabla los criterios utilizados para que otros investigadores puedan tomarla en consideración. Estas categorías de evaluación a su vez permitirán superar una de las limitaciones encontradas en el análisis, que es la falta de referentes y comparaciones con otros estudios de esta temática. Como punto de referencia para el debate se establece que no se debería utilizar el término de evaluación cuando quede reducida a mostrar el resultado de las calificaciones en las experiencias de innovación presentadas. Teniendo esto en cuenta, se puede ver que se hace necesario la evaluación de todo aquello que forma parte del proceso de enseñanza-aprendizaje: alumnado, profesorado, materiales, recursos, metodologías, etc. Pues gracias a ella, se puede ver el valor real de todo. La evaluación no se limita a una descomposición aislada 
de cada elemento. El procedimiento seguido para poder investigar este tema ha sido el de analizar, es decir descomponer en partes. Pero se debe tener presente que la integración de todos los elementos confiere un nuevo sentido a la investigación en la evaluación. Con una nueva legislación educativa en la que se toman en consideración los ODS y en particular en el 4 objetivo, la educación de calidad, se hace imprescindible avanzar en la mejora del proceso evaluador, entre otras razones porque otorga legitimidad a la investigación realizada sobre la enseñanza.

Otro punto para el debate es que no existe educación de calidad sino hay previamente una buena praxis en la investigación educativa en geografía. Por esta razón, cabe destacar la aportación que han realizado todos los autores investigados y que permiten enriquecer desde diferentes aportaciones todos los aspectos tenidos en cuenta.

Por otro lado, a pesar de que la evaluación es un elemento curricular importante, apenas dispone de investigaciones sobre su puesta en práctica. Este hecho también ha quedado reflejado en la revista analizada. En este sentido es significativo que sólo se haya encontrado un artículo sobre evaluación en todo el compendio de números que forman la Segunda Época de la revista Didáctica Geográfica. En consecuencia, se requiere que en un futuro las aportaciones que se realicen sobre materiales, recursos, experiencias, detallen mejor los criterios o argumentos que se utilizan para juzgar, valorar, etc. y aumente la complejidad de los elementos que intervienen y las relaciones que se establecen entre ellos. De esta forma, ha quedado patente la realidad de la evaluación en la revista Didáctica Geográfica y la necesidad de avanzar y profundizar en esta línea de investigación.

En esta aportación que se presenta cabe asumir que existen otros puntos de vista, elementos, procesos, que pueden enriquecer la evaluación. Sin ser exhaustivos, no se ha avanzado en otros aspectos no menos importantes de naturaleza social como los realizados por Fraga, Tonini y Santos de Oliveira (2017).

\section{REFERENCIAS BIBLIOGRÁFICAS}

Bauman, Z. (2013). La cultura en el mundo de la modernidad líquida. Madrid: Fondo de Cultura Económica de España.

Comisión de Mujeres y Ciencia del CSIC. (2020). Informe Mujeres Investigadoras. https://mujeresconciencia.com/2020/07/03/informe-mujeres-investigadorascsic-2020/

Fraga, J., Tonini, I. Mª, Santos de Oliveira, S. (2017). Geografia, diálogos, reflexividades e aproximações. Curitiba (Brasil): Editora CRV. 
García, A. L. (1988). Criterios básicos para la evaluación del aprendizaje de la geografía. Revista de la Facultad de Geografía e Historia, 3, 383-392.

García M., Villar, A., Fraile, P., Sánchez, N. y Márquez, J. (2018). Se hace geografía al andar: la salida de campo itinerante y senderista. Didáctica Geográfica, 19, 103125. https://doi.org/10.21138/DG.418

Gómez-Carrasco, C. J., López-Facal, R. y Rodríguez-Medina, J. (2019). La investigación en Didáctica de las Ciencias Sociales en revistas españolas de Ciencias de la Educación. Un análisis bibliométrico (2007ロ2017). Didáctica de las Ciencias Experimentales y Sociales, 37, 67口88. https://doi.org/10.7203/dces.37.14440

Jerez, O. y Córdoba, M. A. (2016). La producción científica sobre didáctica de la geografía en España y su difusión en el siglo XXI. Revisión de la segunda época de la revista Didáctica Geográfica (1996-2015). In R. Sebastiá y E. M. Tonda (Eds.), La investigación e innovación en la enseñanza de la Geografía (pp. 759780). San Vicente del Raspeig: Universidad de Alicante.

Labarca, R., Barreto, B. y Bernal, J. (2018). Potencialidades geográficas de la Laguna de las Peonías (Venezuela) como museo natural para la enseñanza de la geografía física. Didáctica Geográfica, 19, 127-148. https://doi.org/10.21138/DG.419

Labarca, R. J. y Gouveia, E. L. (2020). Los procesos exogenéticos: una geo-ruta didáctica por la costa occidental del Lago Maracaibo (Venezuela). Didáctica Geográfica, 21, 175-201. https://doi.org/10.21138/DG.498

López, J.A.; Martínez, R. (2016). La cartografía digital y los sistemas de información Geográfica, a través de las publicaciones del grupo de Didáctica de la AGE y la revista Didáctica Geográfica. 613-624. En R. Sebastiá y E. M Tonda, La investigación e innovación en la enseñanza de la Geografía. Alicante: Universidad de Alicante.

López, J. A. y Peral, A. J. (2017). Las vías verdes: escenario para trabajar el medio rural en educación primaria. Didáctica Geográfica, 18, 171-192. https:// didacticageografica.age-geografia.es/index.php/didacticageografica/article/ view/388

Lucendo, A. L. (2016). Propuesta de metodología docente para la adaptación de materias de geografía descriptiva al EEES: el caso de la asignatura de Geografía de Europa. Didáctica Geográfica, 17, 79-100. https://didacticageografica.age-geografia.es/ index.php/didacticageografica/article/view/360

Morote, A. F. (2017). El parque inundable "La Marjal" de Alicante (España) como propuesta didáctica para la interpretación de los espacios de riesgo de inundación. Didáctica Geográfica, 18, 211-230. https://didacticageografica.age-geografia.es/ index.php/didacticageografica/article/view/390 
Morote, A. F. y Hernández, M. (2019). Propuesta de un recurso didáctico para la interpretación del paisaje. La salida de campo en la antigua Laguna de Villena (Alicante). Didáctica Geográfica, 20, 95-121. https://doi.org/10.21138/DG.457

Morote, A.F. (2020) La investigación sobre manuales escolares de Geografía españoles: Análisis bibliométrico (1980-2019). Anales de Geografía de la Universidad Complutense, 40(2), 467-497. http://dx.doi.org/10.5209/AGUC.72983

Najarro, U. y Maroto, J. C. (2019). El desarrollo de las competencias sociales y cívicas en ciencias sociales: itinerario didáctico por los restos fenicios de Almuñécar (Granada). Didáctica Geográfica, 20, 123-152. https://doi.org/10.21138/DG.458

Prieto, J. A., Valera, J. y Gomariz, F. A. (2011). Propuesta de evaluación de la Geografía y de la Historia en el Bachillerato de investigación. En P. Miralles, S. Molina y A. Santisteban (Eds.), La evaluación en el proceso de enseñanza y aprendizaje de las ciencias sociales (403-412). AUPDCS. http://www.didactica-ciencias-sociales. org/publicaciones archivos/2011-murcia-La evaluacion I.pdf

Sande, E. (2005). Simulación y evaluación. Didáctica Geográfica, 7, 497-510. https:// didacticageografica.age-geografia.es/index.php/didacticageografica/article/ view/255

Santos,M.A.(1988). Patología general de la evaluación educativa.Infancia y Aprendizaje, 41, 143-158. https://dialnet.unirioja.es/servlet/articulo?codigo=48299

Sebastiá, R. y Tonda, E. M. (2011). Características y evolución de la revista Didáctica Geográfica. Didáctica Geográfica, 12, 19-48. https://didacticageografica.agegeografia.es/index.php/didacticageografica/article/view/57

Sebastiá, R. y Tonda, E. M. (2014). Didáctica de la Geografía en la revista Enseñanza de las Ciencias Sociales: Análisis bibliométrico y definición de las principales vías de investigación. Enseñanza de las Ciencias Sociales, 13, 107-116. https://www. raco.cat/index.php/EnsenanzaCS/article/view/285780

Sebastiá, R. y Tonda, E. M. (2015). El concepto del espacio geográfico en la enseñanza de la Geografía en los niveles educativos no universitarios. Análisis bibliométrico. In J. de la Riva, P. Ibarra, R. Montorio, y M. Rodrigues (Eds.), Análisis espacial y representación geográfica: innovación y aplicación, (pp. 1505-1514). Zaragoza: Universidad de Zaragoza-AGE.

Sebastiá, R. y Tonda, E. M. (2016). Evaluación de recursos didácticos TIC: Lecciones de orientación espacial en "mi amiga la tierra", Instituto Geográfico Nacional (España). In L. Alanís, J. Almuedo, G. De Oliveira, R. Iglesias y B. Pedregal (Coords.), Nativos digitales y geografía en el siglo XXI: Educación geográfica y 
sistemas de aprendizaje (pp. 718-729). Madrid: Grupo de didáctica de la Geografía de la Asociación de Geógrafos Españoles.

Valera, F. J., Gomariz, F. y Prieto, J. A. (2011). Hacia una evaluación de la Geografía en Educación Secundaria: la aplicación de los principios del método geográfico y las competencias básicas. En P. Miralles, S. Molina y A. Santisteban (Eds.), La evaluación en el proceso de enseñanza y aprendizaje de las ciencias sociales (461-470). AUPDCS. http://www.didactica-ciencias-sociales.org/publicaciones archivos/2011-murcia-La evaluacion I.pdf 